\title{
The molecular profile of luminal B breast cancer
}

\author{
This article was published in the following Dove Press journal: \\ Biologics:Targets and Therapy \\ 23 August 2012 \\ Number of times this article has been viewed
}

\section{Chad J Creighton \\ Department of Medicine and Dan L Duncan Cancer Center Division of Biostatistics. Baylor College of Medicine, Houston, TX, USA}

Correspondence: Chad J Creighton Dan L Duncan Cancer Center Division of Biostatistics, Baylor College of Medicine, One Baylor Plaza MS 305, Houston, TX 77030, USA

$\mathrm{Tel}+\mathrm{I} 7 \mid 37982264$

Fax + I 7I379827I6

Email creighto@bcm.edu

\begin{abstract}
Molecular profiling studies have found that estrogen receptor-positive (ER+) human breast cancers are comprised of at least two distinct diseases with differing biologies. With the advent of DNA microarrays, global gene expression patterns were used to define the luminal A and luminal B subtypes of ER+ breast cancer, with luminal B cancers showing a more aggressive phenotype including substantially worse outcomes in patients. The luminal B subtype designation could be considered a surrogate for those ER+ tumors having low progesterone receptors, high proliferation, high grade, and predicted poor response to hormone therapy. While they express estrogen receptors, luminal B cancers do not show a corresponding expression of estrogenregulated genes, and may therefore rely upon alternative pathways for growth. At the molecular level, luminal B cancers appear dramatically distinct from luminal A cancers, at the levels of gene expression, gene copy, somatic mutation, and DNA methylation; luminal B cancers are also genetically and genomically altered to a greater extent than luminal A cancers. While, in the clinical setting, luminal B is typically regarded as an ER+, hormone-sensitive disease, more research is needed into how to better treat it. Comprehensive profiling initiatives, such as The Cancer Genome Atlas, have recently provided us a catalog of mutated or copy altered genes, from which new therapeutic targets could potentially be mined. Candidate pathways that might be targeted in luminal B include those involving growth factor receptors, including HER2 and EGFR, as well as PI3K/Akt/mTor.
\end{abstract}

Keywords: luminal B, molecular profiling, integrative analysis, breast cancer, TCGA

\section{Introduction}

Breast cancer encompasses a number of different diseases. In the clinical setting, breast cancers by convention are subdivided according to estrogen receptor (ER) and HER2 status; ER-positive (ER+) tumors typically respond to hormone therapy, while HER2 + tumors respond to anti-HER2 therapy, and no targeted therapy is currently in widespread use for ER-/HER- tumors. Progesterone receptor (PR) is another molecular marker that may be used in the clinic, as loss of PR in ER+ tumors is thought to be predictive for lack of response to hormone therapy. ${ }^{1}$ Over a decade ago, gene expression profiling (at the mRNA level) uncovered distinct molecular subtypes of breast cancer, each defined by a large number of genes. ${ }^{2-4}$ These subtypes - which include basal-like, HER2-enriched, normal-like, luminal A, and luminal B - largely reflect the established clinical- and histological-based classifications, with basal-like representing ER-/HER2- cancers, HER2-enriched representing ER-/HER2+, and normal-like and luminal A/B subtypes representing ER+. One notable finding from the gene expression studies was that luminal B cancers showed worse outcomes as 
compared to luminal A cancers, ${ }^{2}$ which indicated that ER+ breast cancer itself may be made up of at least two distinct diseases with differing biology.

The luminal B subtype, understood to be the more aggressive form of ER+ breast cancer, is the subject of this review. Here we will broadly describe luminal B breast cancer in terms of its molecular profile, making use of public datasets including those from The Cancer Genome Atlas (TCGA). ${ }^{5-7}$ The premise put forth here is that luminal B cancers represent a unique subtype of breast cancer, with a distinctive biology from that of luminal A cancers, rather than simply representing a more advanced or aggressive form of luminal A.

\section{Pathophysiology of luminal B breast cancer}

Though the designation of luminal B first came about with the early gene expression profiling studies of breast cancer near the start of the millennium, it has long been understood that there exists a more aggressive form of ER+ breast cancer. Earlier studies had noted that $\sim 30 \%$ of ER+ invasive breast cancers showed no benefit from hormone therapy, suggesting that these cancers either augment or entirely bypass the classical estrogen-stimulated mitogenic pathway. ${ }^{8}$ Markers predicting hormone therapy response within ER+ cancers have been sought, the most notable of these being PR. Loss of PR expression is thought to represent a surrogate for a more aggressive disease phenotype that is less dependent upon estrogen signaling; in correlative studies, PR loss has been associated with lower ER levels, more positive nodes, aneuploidy, larger tumor size, higher proliferation, and expression of growth factor receptors (GFRs) including EGFR and HER2. ${ }^{1}$

The advent of DNA microarrays made it possible to profile breast cancers for mRNA expression of thousands of genes. Early profiling studies took a fresh approach to classifying breast cancer, allowing the expression data themselves to segregate human breast tumors, rather than relying on prior biological knowledge and possible biases. Expression profiling first defined five intrinsic molecular subtypes of breast cancer: basal-like, HER2-enriched, normal-like, luminal A, and luminal B. A sixth subtype, claudin-low, was discovered later, perhaps due to its greater rarity., ${ }^{9,10}$ Other additional ERsubtypes could include a "molecular apocrine" subtype (ER-/ HER2+ but with activation of androgen receptor signaling), and an "interferon" subtype (with high expression of interferon regulated-genes). ${ }^{11}$ Recently, there have been some suggestions $^{12,13}$ that the normal-like breast cancer might be a mere artifact of sample representation (ie, disproportionately high content of normal tissue contamination), though such concerns would not extend to the other subtypes, including luminal B.

As noted above, the mRNA-based classifications, as originally defined by Perou and colleagues, largely reflected the histological-based classifications. The luminal and normal-like subtypes reflect the ER+ form of breast cancer. The designation of luminal was derived due to the finding that these cancers show mRNA and protein expression of keratins $8 / 18,{ }^{3}$ typically associated with luminal epithelial cells (as opposed to basal cells which express keratins 5/6). The luminal subtype of breast cancer was further subdivided into A and B groups, as luminal B showed widespread expression differences as well as substantially worse outcomes. ${ }^{2}$ In the future, as larger cohorts of breast tumors are profiled at multiple data levels, the molecular designations of breast cancer may be further refined.

While the intrinsic molecular subtypes of breast cancer have been defined and examined on the basis of hundreds of genes, ${ }^{2,3,9,14}$ in the interests of a more focused gene list for the purposes of a clinical assay, the PAM50 assay was developed, which consists of 50 genes by which breast cancers can be classified by mRNA-based subtype. ${ }^{13,15,16}$ Figure 1A illustrates the patterns of the PAM50 genes, both in an expression profile dataset $(\mathrm{N}=240)$ from the research group which first developed the assay, ${ }^{14}$ and in a compendium of nine separate datasets $^{17-25}$ profiled on the same assay platform $(\mathrm{N}=1340$, previously collected by Kessler et al). ${ }^{26}$ It is notable how the PAM50 gene patterns are remarkably consistent between the two datasets. It is also evident that luminal B shares some gene patterns with luminal A (eg, ER genes ESR1, FOXA1, and $B C L 2)$ and some patterns with basal-like including expression of proliferation markers (eg, Ki-67 gene MKI67, survivin gene BIRC5, and cyclin B1 gene). It has been put forth that luminal B might represent the ER+/HER2+ form of breast cancer; ${ }^{4}$ however, in our compendium dataset, HER2 gene $E R B B 2$ does not show high levels in luminal B (Figure 1A).

Within the compendium dataset, ${ }^{26}$ as reported elsewhere, luminal B cancers have much worse outcomes compared to luminal A, the outcomes of luminal B being comparable to, if not worse than that of the basal-like and HER2-enriched subtypes (Figure 1B). Between different patient cohorts, the relative outcomes of the intrinsic mRNA-based subtypes may vary; for example, the HER2-enriched subtype may have a better outcome in patients treated with anti-HER2 therapy, ${ }^{27,28}$ and in at least one early study using a small cohort, normallike breast cancers actually showed a worse outcome comparable to that of luminal B. ${ }^{2}$ However, luminal B has been 


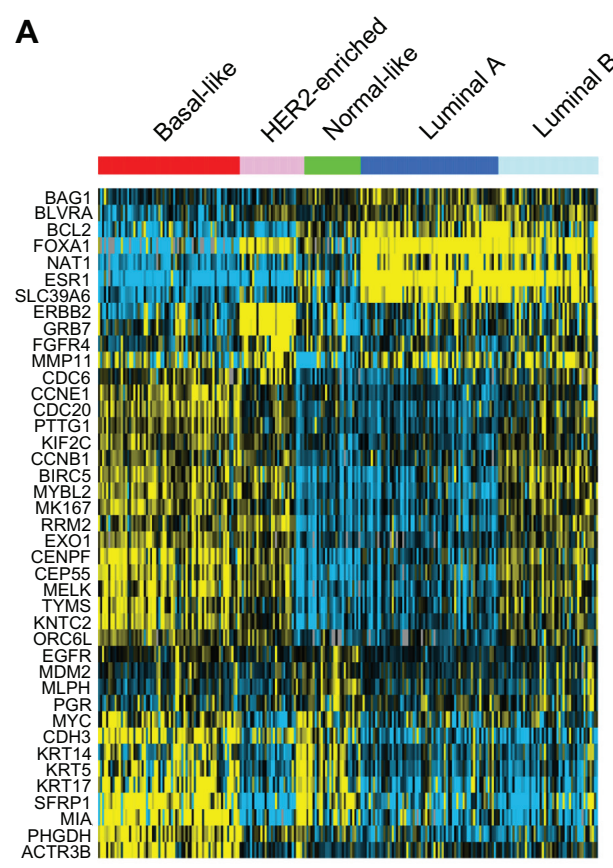

Hoadley et al dataset $(\mathrm{N}=\mathbf{2 4 0})$

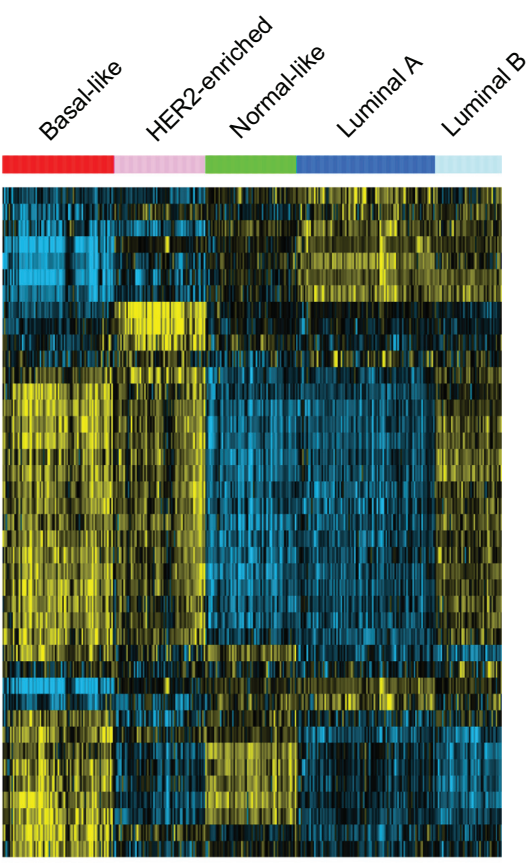

U133A tumor compendium dataset $(N=1340)$

Gene expression Low High

B

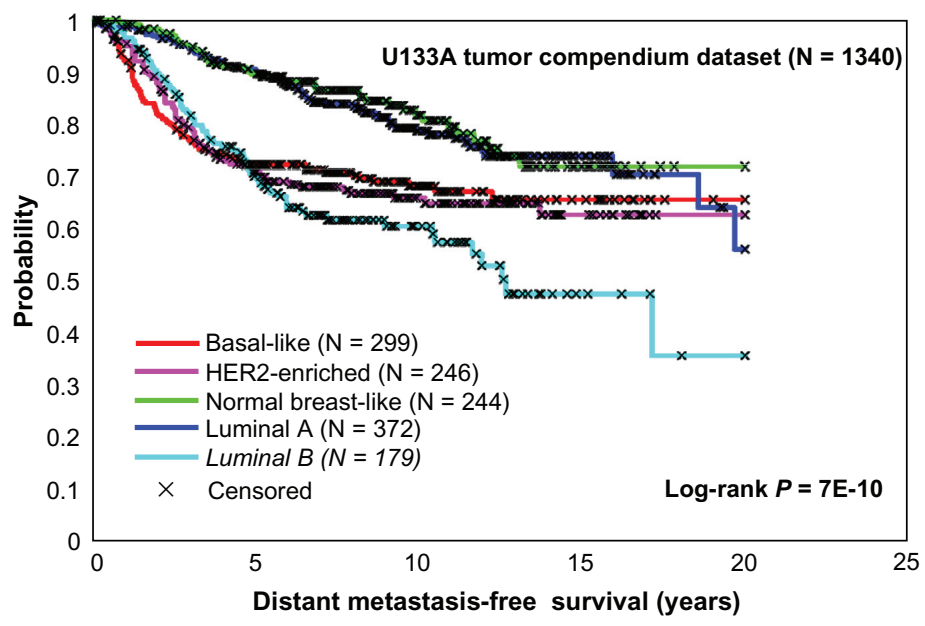

Figure I Luminal B breast cancer is associated with substantially worse patient outcomes. (A) Expression patterns of the PAM50 gene set ${ }^{13}$ ( 4 I genes represented on the UI33A array platform) in the mRNA profile datasets from Hoadley et al, ${ }^{14}(\mathrm{~N}=247)$ and Kessler et al. ${ }^{26} \mathrm{UI} 33 \mathrm{~A}$ compendium ( $\mathrm{N}=1340$, representing nine separate studies). Using the PAM50 genes and the Hoadley subtype assignments, inter-profile correlations between the Hoadley and Kessler datasets were used to assign mRNA-based subtypes (basal-like, HER2-enriched, normal-like, luminal A, luminal B) to the Kessler profiles. (B) Kaplan-Meier plot of distant metastasis-free survivals for the mRNA-based subtypes. Survival capped at 20 years.

found to consistently show poor outcomes in studies using sizable datasets, including patients receiving antiestrogen therapy (such as the Loi et al dataset, ${ }^{21}$ which is represented in our compendium).

\section{Diagnosis of luminal B breast cancer}

Assays for diagnosing luminal $\mathrm{B}$ breast cancer in the clinic are currently available, though it will likely take some time for these assays to become routine, as new clinical practices are often gradually adopted. PR is one marker that is frequently measured in the clinic along with ER, in order to further subdivide ER+ breast cancers by prognosis or anticipated therapeutic response, ${ }^{1,28-31}$ though the association of PR with hormone response has not been observed everywhere, ${ }^{32,33}$ which may be an indicator of a single biomarker having insufficient information as compared to a biomarker panel. Clinical variables such as grade or Ki-67 can help distinguish the subset of ER+ breast tumors with expected worse outcomes. ${ }^{34}$ 
The PAM50 assay, ${ }^{13,15,16}$ which classifies breast cancer by mRNA-based subtype, is compatible with formalin-fixed, paraffin-embedded-derived RNA, which facilitates its use in the clinic; the assay is currently being developed for clinical use on the NanoString nCounterTM Analysis System (Nanostring Technologies, Seattle, WA) and is also offered as a laboratory-developed test, using a qualitative reversetranscription polymerase chain reaction format from ARUP Laboratories (Salt Lake City, UT). ${ }^{15}$

While numerous prognostic gene signatures of breast cancer have been developed using expression profiling, it is understood that the different signatures by and large work by differentiating luminal A breast cancers from all other subtypes including luminal B. ${ }^{15,35}$ In addition to PAM50, other gene assays for predicting the outcome of ER+ breast cancer include Oncotype DX (Genomic Health, Redwood City, CA) and MammaPrint (Agendia, Amsterdam, The Netherlands). Oncotype DX, based on 16 cancer-related genes, ${ }^{36}$ is perhaps the most widely used clinical gene-expression assay in the USA, ${ }^{15}$ while MammaPrint is based on 70 genes ${ }^{37}$ Similar to what has been observed elsewhere using other datasets, ${ }^{35,38,39}$ in the Kessler U133A compendium breast tumor dataset, luminal B tumors have lower PR mRNA levels and predicted worse outcomes according to either Oncotype DX or MammaPrint signatures (Figure 2). Therefore, there are a number of ways the luminal B or poor prognostic subset of $\mathrm{ER}+$ breast cancer may be defined in the clinical setting. While the various assays may rely on different prognostic gene sets, the genes all appear to point to an overall biological phenomenon, likely involving hundreds if not thousands of genes, of which a handful may be represented in a given assay.

\section{Current and emerging novel targets and therapies}

As luminal B breast cancer expresses ER, the estrogen pathway is regarded as a therapeutic target for this disease, though the case could readily be made that additional targets are needed. According to 2011 St Gallen consensus recommendations, where luminal $\mathrm{B}$ could be defined in the clinical setting as ER+/HER2-/Ki67+, standard treatment would include endocrine therapy with or without cytotoxic therapy. ${ }^{15,40}$ However, a large percentage of ER+ breast cancers, which may be regarded as representing luminal $\mathrm{B}$, do not respond to endocrine therapy. ${ }^{8,41}$ Where PR (under-expressed in luminal B) has long been studied as a marker of functional ER, therapeutic targets for ER+/ PR- disease have been considered. ${ }^{1}$ Studies suggest that estrogen-independent disease represents a switch from using the estrogen pathway to using an alternative GFR signaling pathway; such candidates including HER2, EGFR, IGF, and Akt/PI3K/mTOR. ${ }^{42-46}$

Molecular profiling is one means of identifying novel therapeutic candidates for diseases such as luminal B breast cancer, and to this end, the TCGA initiative is currently engaged in profiling human cancers at multiple levels of molecular complexity, including mRNA, protein (by RPPA assay), microRNA, gene promoter methylation, DNA copy, and somatic mutation. ${ }^{5-7}$ TCGA recently completed a study of breast cancer, ${ }^{7}$ which provided a great deal of insight into the molecular profile of luminal B as well as the other intrinsic subtypes. In this review, we will discuss findings of the TCGA study particularly relevant to luminal B (as all TCGA tumors were subtyped according to their mRNA profile), providing some views of the associated data as well, which are all public. ${ }^{7,47}$

The TCGA study results further demonstrated that luminal B appears quite distinct from luminal $\mathrm{A}$ at the molecular level, reinforcing the notion that the two subtypes represent different diseases. For one thing, as the mRNAbased subtypes were originally defined in an unsupervised fashion, allowing the mRNA profile data to group tumors, the same exercise was also carried out to define subtypes

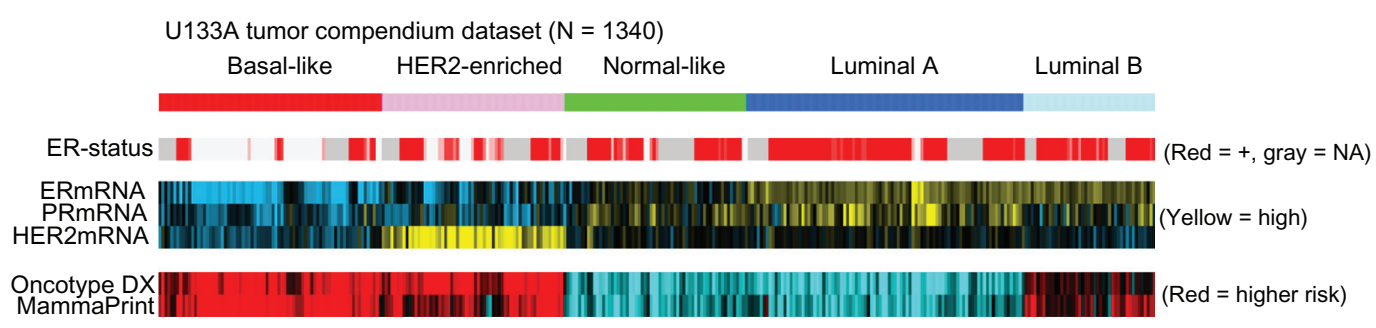

Figure 2 The luminal B subtype represents both ER+ breast cancer with low PR levels and ER+ breast cancer with predicted worse outcome.

Notes: For tumor profiles in the Kessler compendium, corresponding mRNA levels for ER, PR, and HER2 are shown. In addition, the tumors were scored for predicted outcome, using the genes in the OncotypeDX ${ }^{36}$ and MammaPrint ${ }^{37}$ diagnostic assays (scores computed by adding the normalized values for the positively weighted genes and subtracting the values for the negatively weighted genes).

Abbreviations: ER+ estrogen receptor-positive; PR, progesterone receptor; ER, estrogen receptor. 
at each level of microRNA, methylation, and protein; the mRNA-based subtypes were found to be reflected in the other molecular data types, ${ }^{7}$ and those results are represented here in a different format (Figure 3) to highlight the finding that the mRNA-based luminal B subtype overlaps highly with specific subtypes at the methylation and protein levels (though interestingly not at the microRNA level), showing

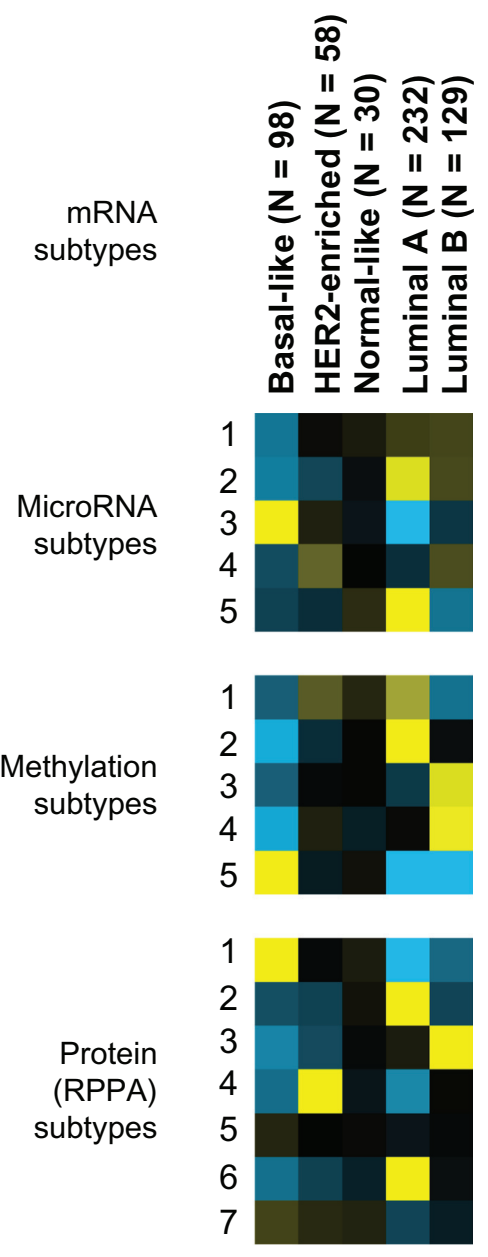

\section{Fisher's exact z-score

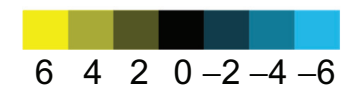

Enriched

Anti-enriched

Figure 3 The (mRNA-based) luminal B breast cancer subtype is reflected in alternative classifications of breast cancer, based on molecular profiling at levels of microRNA, methylation, and protein.

Notes: Data are from TCGA of 547 human breast tumors profiled for gene expression and subtyped for mRNA-based classification. Most all of these tumors were profiled on other platforms for microRNA, DNA methylation, and protein (RPPA). By each of the other platforms, unsupervised clustering classified the tumors into distinct subtypes; more detail on these alterative classifications is provided in the TCGA breast study. ${ }^{7}$ Shown here is the significance of overlap between each of the mRNA-based subtypes and the subtypes based on the other platforms (bright yellow, highly significant enrichment, with \pm 2 roughly corresponding to a nominal two-sided significance of $P<0.05$ ).

Abbreviations: TCGA, The Cancer Genome Atlas; RPPA, reverse-phase protein array. patterns distinct from those of luminal A. Furthermore, luminal B cancers were somatically mutated and copy altered to a significantly greater extent than luminal A cancers, at levels comparable to what was observable in basal-like and HER2-enriched cancers (Figure 4), consistent with previous findings. ${ }^{17,39}$

The TCGA study has yielded a catalog of genes that are genetically or genomically altered in luminal B cancers, and many of these aberrations could have essential roles in the disease phenotype; the nontrivial challenge, however, would be in our being able to distinguish the driving alterations from those that are simply passengers. In terms of the mutation landscape, PIK3CA and TP53 are the most frequent somatic targets in breast cancer, as well as in the luminal B subset (Figure 5). Numerous other genes appear recurrently mutated at a much lower frequency, and many

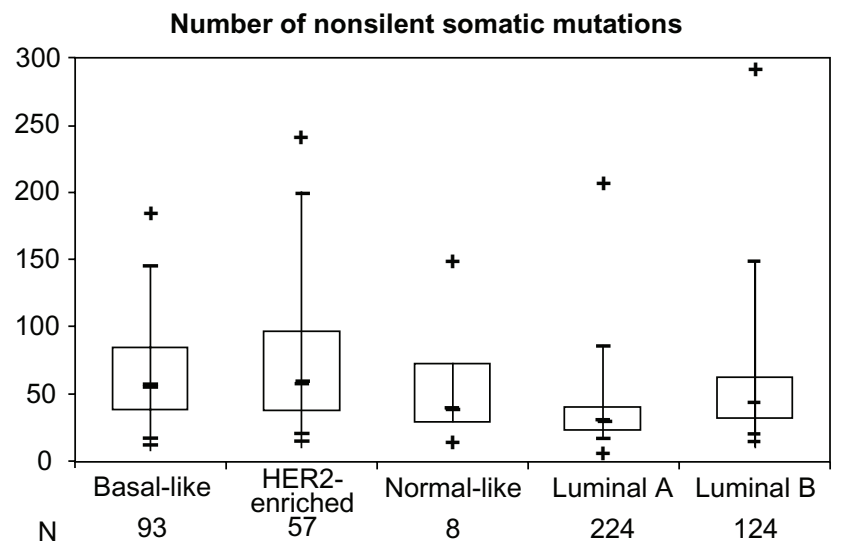

Copy number variation index

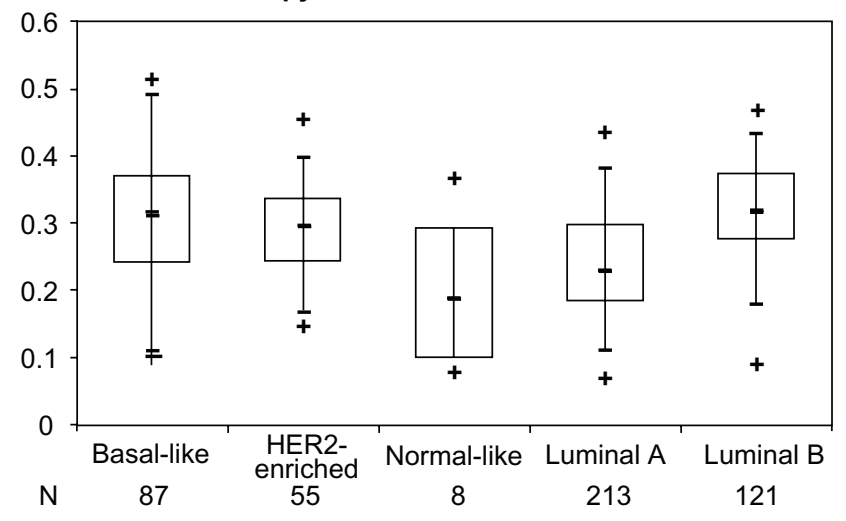

Figure 4 Luminal B cancers are genetically and genomically altered to a greater extent than luminal A cancers (and comparable to the ER-subtypes).

Notes: Top panel shows box plot for the numbers of nonsilent somatic mutations for each mRNA-based subtype. Bottom panel shows box plot of the variation from normal copy levels for each subtype (taking the standard deviation across all genes in the profile of the tumor:normal log-ratio values). Data are from TCGA ${ }^{7}$ (top panel, $\mathrm{N}=506$ tumors with somatic mutation and expression data; bottom panel, $\mathrm{N}=484$ tumors with mutation, expression, and LOH data).

Abbreviations: ER-, estrogen receptor-negative; TCGA, The Cancer Genome Atlas; LOH, loss of heterozygosity. 

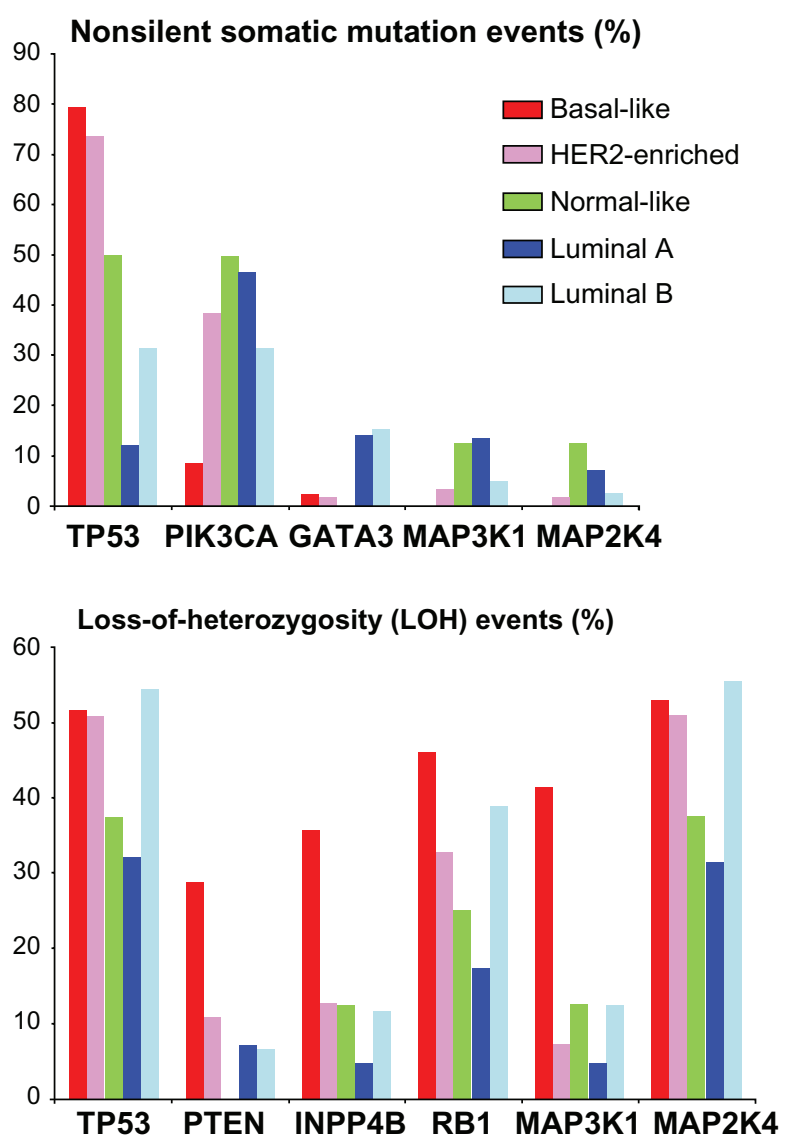

Figure 5 Luminal B cancers have distinct patterns of alterations in genes impacting key pathways including $\mathrm{P} 53, \mathrm{PI} 3 \mathrm{~K}, \mathrm{Rb}$, and MAP kinase.

Notes: For selected genes, the percentage of tumors, by mRNA-based subtype, altered by somatic mutation (top panel) or by $\mathrm{LOH}$ (bottom panel). Data are from TCGA $^{7}$ (top panel, $\mathrm{N}=506$ tumors with somatic mutation and expression data; bottom panel, $\mathrm{N}=484$ tumors with mutation, expression, and LOH data).

Abbreviations: LOH, loss of heterozygosity; TCGA, The Cancer Genome Atlas.

of these could be found to have important roles in at least a subset of luminal B cancers. In terms of the copy landscape, there is a very large number of genes amplified or deleted in luminal $\mathrm{B}$, the complete list being too large to enumerate here (but available in the TCGA report). ${ }^{7}$ Copy loss events that show differential patterns among the subtypes include those involving TP53, PTEN, INPP4B, RB1, MAP3K1, and $M A P 2 K 4$ (Figure 5).

\section{The origin of luminal B breast cancer}

Observations and unanswered questions as to the origin of luminal B breast cancers may follow a similar line of reasoning regarding the origin of ER- breast cancer. ${ }^{8}$ Luminal B cancers, though ER+, are resistant to hormone therapy and have a distinct molecular phenotype from hormone-sensitive luminal A cancers, with luminal B sharing many molecular features with ER- cancer subtypes (Figures 1A and 5).
One important question is whether luminal B cancer/cancer precursors evolve from luminal A cancer/cancer precursors, or have a path of initiation and progression that is completely distinct from luminal A; these two alternate hypotheses are represented graphically in Figure 6, though these are only conceptual, and the truth may well lie somewhere in between the two.

Some observations may suggest that luminal B and other hormone resistant breast cancer subtypes evolve from luminal A cancer or a precursor to luminal A. For example, ER- breast cancers (and by implication luminal B) are believed to evolve from ER+ precursors or cells. ${ }^{8}$ Estrogen dynamically regulates several factors and is regulated by others, and loss of ER function and increase of GFR may well occur during the progression of breast cancer to a hormone-resistant state. ${ }^{1}$ Cell line models of ER+, hormone-sensitive (ie, luminal A-like) cancers can be manipulated experimentally into switching to use of alternative GFR signaling pathways, often resulting in downregulation of ER or PR or both, ${ }^{38,48,49}$ and conversely some ER- cancers can be made to express ER and become hormone-sensitive; ${ }^{50}$ this would suggest that many breast cancers at least are not genetically hard-wired for dependence upon a single pathway, but may evolve from one pathway to another. Additionally, we see that, compared to luminal A, luminal B cancers have significantly greater numbers of

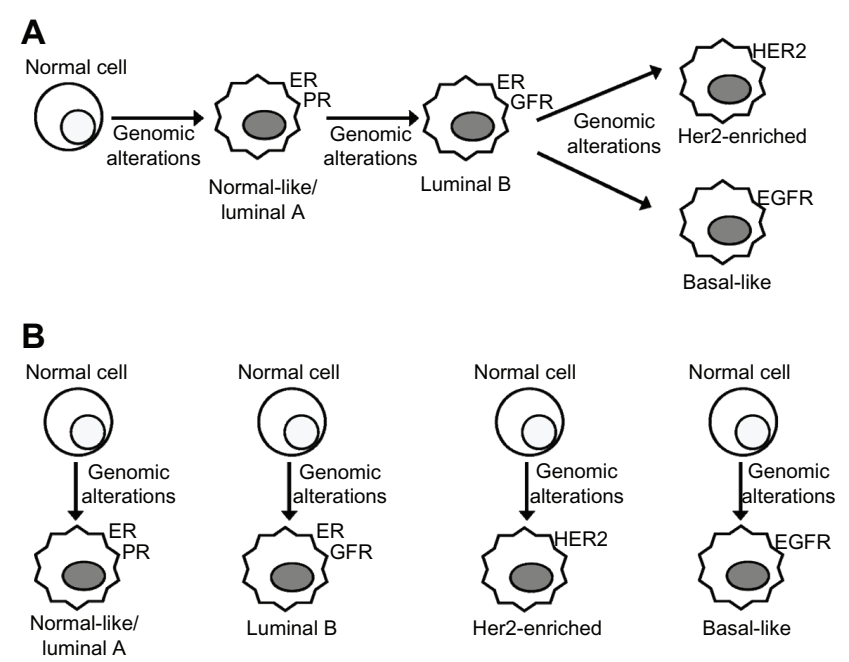

Figure 6 Two different models to explain the possible origin of the breast cancer subtypes (including luminal B). (A) "Linear evolution" model, whereby cancer cells or cancer precursor cells gradually evolve (by accumulation of genomic alterations) from a luminal $A$ or normal-like subtype (with ER/PR expression), to a luminal $B$ subtype (with loss of PR and possible gain of growth factor receptor, or GFR signaling), to either a HER2-enriched or Basal-like subtype (with complete loss of ER signaling and increase of GFR signaling). (B) "Distinct pathways of progression" model, whereby each breast cancer subtype follows a path of initiation and progression that is independent of that of the other subtypes.

Abbreviations: ER, estrogen receptor; PR, progesterone receptor; GFR, growth factor receptor. 
mutations and copy number alterations (Figure 4), consistent with the idea of a cancer cell acquiring alterations in the path from a luminal A to a luminal B state.

Alternatively, other observations can suggest that luminal B and the other breast cancer subtypes develop and evolve independently of each other. The decision for a cell to be hormone-independent versus hormone-dependent likely happens early on, before the transition to an invasive breast cancer cell; breast cancers are understood to evolve in a nonobligatory fashion through an increasingly abnormal series of hyperplasias, atypical hyperplasias, and noninvasive or in situ carcinomas, with the earlier precursor cells being nearly all ER+ and the later (pre-invasive) precursor cells having a much greater proportion of ER- cells. ${ }^{8}$ Furthermore, when examining gene expression profiles of primary tumors and distant metastases from the same patients, one study found that the subtype associations were preserved throughout the metastatic process of breast cancer. ${ }^{51}$

At the molecular level, the various subtypes appear quite distinct from each other, making it difficult to trace a clear path of genomic alterations leading from one subtype to another. For example, while luminal B may appear genomically altered to a similar extent to that of basal-like (Figure 4), the copy profile of basal-like dramatically differs from that of any of the other subtypes, with basal-like sharing extensive copy and transcriptional similarities with serous ovarian cancers. $^{7}$ DNA methylation profiling ${ }^{7}$ associates specific patterns of epigenetic hardwiring with luminal B cancers, which appear quite distinct to that associated with luminal A or basal-like cancers (Figure 3 ). When individual genes are considered, we find for example, that somatic mutations in $P I K 3 C A$ are most frequent in luminal A cancers (46\%), less frequent in luminal B (31\%), and least frequent in basal-like cancers ( $9 \%$, Figure 5); this would seem counter to the notion of luminal B or basal-like cancers having originally started from a luminal A genetic profile. Similarly, other studies, such as ones comparing molecular features of high-grade (eg, luminal B or basal-like) versus low-grade breast cancers, have concluded that a clear path of progression between the two would be difficult to define. ${ }^{52}$

\section{Clinical potential and future research}

At present, luminal B breast cancer is routinely treated as an ER+, hormone-sensitive disease. ${ }^{15,40}$ While often considered as simply a more aggressive form of the ER+ subtype, luminal $\mathrm{B}$ might be considered more in the future as a type of breast cancer that is entirely distinct in many ways from luminal A.
While triple-negative (ER-/PR-/HER2-) breast cancer is of particular interest to the research community, owing to there being as yet no systematic targeted therapy for this disease, the same level of effort might be directed as well towards identifying new therapies for luminal B cancers. As luminal B cancers appear to rely less on the estrogen pathway, recent clinical trial studies have considered targeting alternate pathways in advanced stage ER+ cancer, include EGFR (eg, using the drug gefitinib) ${ }^{42}$ and PI3K/Akt/mTOR (eg, using everolimus). ${ }^{43}$ The recent BOLERO-2 clinical trial results showed progression-free survival in patients with hormone refractory ER+ disease being substantially prolonged by mTOR inhibitor everolimus. ${ }^{43}$ While results such as these are encouraging, more study is needed to assess the efficacy of such drugs in the adjuvant setting.

\section{Conclusion and future perspective}

Over the years, comprehensive molecular profiling of breast cancer has deepened our understanding of luminal B breast cancer as a unique disease, distinct from either luminal $\mathrm{A}$ or ER- cancers. The recent TCGA study and others have provided an extensive catalog of the genetic and genomic aberrations associated with luminal B, and the nontrivial task remains of distinguishing those aberrations that are simply correlative from those that have a driving role in the disease phenotype. Better therapeutic approaches and targets are needed for a large subset of ER+ tumors that might initially have been deemed treatable, and the molecular profile of luminal B may well provide additional clues needed to tackle this important challenge.

\section{Acknowledgments}

The author is supported in part by NIH/NCI grants P30 CA125123, P50 CA58183-16, and U24CA143843.

\section{Disclosure}

The author reports no conflicts of interest in this work.

\section{References}

1. Cui X, Schiff R, Arpino G, Osborne CK, Lee AV. Biology of progesterone receptor loss in breast cancer and its implications for endocrine therapy. J Clin Oncol. 2005;23(30):7721-7735.

2. Sørlie T, Perou CM, Tibshirani R, et al. Gene expression patterns of breast carcinomas distinguish tumor subclasses with clinical implications. Proc Natl Acad Sci U S A. 2001;98(19):10869-10874.

3. Perou CM, Sørlie T, Eisen MB, et al. Molecular portraits of human breast tumours. Nature. 2000;406(6797):747-752.

4. Prat A, Perou C. Deconstructing the molecular portraits of breast cancer. Mol Oncol. 2011;5(1):5-23.

5. Cancer Genome Atlas Research Network. Comprehensive genomic characterization defines human glioblastoma genes and core pathways. Nature. 2008;455(7216):1061-1068. 
6. Cancer Genome Atlas Research Network. Integrated genomic analyses of ovarian carcinoma. Nature. 2011;474(7353):609-615.

7. Cancer Genome Atlas Research Network. Comprehensive molecular portraits of human breast tumors. Nature. In press 2012.

8. Allred DC, Brown P, Medina D. The origins of estrogen receptor alphapositive and estrogen receptor alpha-negative human breast cancer. Breast Cancer Res. 2004;6(6):240-245.

9. Herschkowitz JI, Simin K, Weigman VJ, et al. Identification of conserved gene expression features between murine mammary carcinoma models and human breast tumors. Genome Biol. 2007;8(5):R76.

10. Creighton CJ, Li X, Landis M, et al. Residual breast cancers after conventional therapy display mesenchymal as well as tumor-initiating features. Proc Natl Acad Sci U S A. 2009;106(33):13820-13825.

11. Farmer P, Bonnefoi H, Becette V, et al. Identification of molecular apocrine breast tumours by microarray analysis. Oncogene. 2005;24(29):4660-4671.

12. Peppercorn J, Perou CM, Carey LA. Molecular subtypes in breast cancer evaluation and management: divide and conquer. Cancer Invest. 2008;26(1):1-10.

13. Parker JS, Mullins M, Cheang MC, et al. Supervised risk predictor of breast cancer based on intrinsic subtypes. J Clin Oncol. 2009;27(8):1160-1167.

14. Hoadley KA, Weigman VJ, Fan C, et al. EGFR associated expression profiles vary with breast tumor subtype. BMC Genomics. 2007;8:258.

15. Prat A, Ellis MJ, Perou CM. Practical implications of gene-expressionbased assays for breast oncologists. Nat Rev Clin Oncol. 2011;9(1): $48-57$.

16. Nielsen TO, Parker JS, Leung S, et al. A comparison of PAM50 intrinsic subtyping with immunohistochemistry and clinical prognostic factors in tamoxifen-treated estrogen receptor-positive breast cancer. Clin Cancer Res. 2010;16(21):5222-5232.

17. Chin K, DeVries S, Fridlyand J, et al. Genomic and transcriptional aberrations linked to breast cancer pathophysiologies. Cancer Cell. 2006;10(6):529-541.

18. Miller LD, Smeds J, George J, et al. An expression signature for p53 status in human breast cancer predicts mutation status, transcriptional effects, and patient survival. Proc Natl Acad Sci U S A. 2005;102(38):13550-13555.

19. Desmedt C, Piette F, Loi S, et al. Strong time dependence of the 76-gene prognostic signature for node-negative breast cancer patients in the TRANSBIG multicenter independent validation series. Clin Cancer Res. 2007;13(11):3207-3214.

20. Wang Y, Klijn JG, Zhang Y, et al. Gene-expression profiles to predict distant metastasis of lymph-node-negative primary breast cancer. Lancet. 2005;365(9460):671-679.

21. Loi S, Haibe-Kains B, Desmedt C, et al. Definition of clinically distinct molecular subtypes in estrogen receptor-positive breast carcinomas through genomic grade. J Clin Oncol. 2007;25(10):1239-1246.

22. Schmidt MB, Böhm D, von Törne $C$, et al. The humoral immune system has a key prognostic impact in node-negative breast cancer. Cancer Res. 2008;68(13):5405-5413.

23. Zhang Y, Sieuwerts AM, McGreevy M, et al. The 76-gene signature defines high-risk patients that benefit from adjuvant tamoxifen therapy. Breast Cancer Res Treat. 2009;116(2):303-309.

24. Minn AJ, Gupta GP, Siegel PM, et al. Genes that mediate breast cancer metastasis to lung. Nature. 2005;436(7050):518-524.

25. Minn AJ, Gupta GP, Padua D, et al. Lung metastasis genes couple breast tumor size and metastatic spread. Proc Natl Acad Sci U S A. 2007;104(16):6740-6745.

26. Kessler JD, Kahle KT, Sun T, et al. A SUMOylation-dependent transcriptional subprogram is required for Myc-driven tumorigenesis. Science. 2012;335(6066):348-353.

27. Baselga J. Treatment of HER2-overexpressing breast cancer. Ann Oncol. 2010;21 Suppl 7:vii36-vii40.

28. Oldenhuis CN, Oosting SF, Gietema JA, de Vries EG. Prognostic versus predictive value of biomarkers in oncology. Eur $J$ Cancer. 2008;44(7):946-953.
29. Bardou VJ, Arpino G, Elledge RM, Osborne CK, Clark GM. Progesterone receptor status significantly improves outcome prediction over estrogen receptor status alone for adjuvant endocrine therapy in two large breast cancer databases. J Clin Oncol. 2003;21(10): 1973-1979.

30. Arpino G, Weiss H, Lee AV, et al. Estrogen receptor-positive, progesterone receptor-negative breast cancer: association with growth factor receptor expression and tamoxifen resistance. J Natl Cancer Inst. 2005;97(17):1254-1261.

31. Osborne CK, Yochmowitz MG, Knight WA 3rd, McGuire WL. The value of estrogen and progesterone receptors in the treatment of breast cancer. Cancer. 1980;46(Suppl 12):2884-2888.

32. Early Breast Cancer Trialists' Collaborative Group (EBCTCG), Davies C, Godwin J, et al. Relevance of breast cancer hormone receptors and other factors to the efficacy of adjuvant tamoxifen: patientlevel meta-analysis of randomised trials. Lancet. 2011;378(9793): 771-784.

33. Early Breast Cancer Trialists' Collaborative Group. Systemic treatment of early breast cancer by hormonal, cytotoxic, or immune therapy. 133 randomised trials involving 31,000 recurrences and 24,000 deaths among 75,000 women. Lancet. 1992;339(8785):71-85.

34. Cheang MC, Chia SK, Voduc D, et al. Ki67 index, HER2 status, and prognosis of patients with luminal B breast cancer. J Natl Cancer Inst. 2009;101(10):736-750.

35. Fan C, Oh DS, Wessels L, et al. Concordance among gene-expressionbased predictors for breast cancer. N Engl J Med. 2006;355(6): 560-569.

36. Paik S, Shak S, Tang G, et al. A multigene assay to predict recurrence of tamoxifen-treated, node-negative breast cancer. $N$ Engl $J$ Med. 2004;351(27):2817-2826.

37. van 't Veer LJ, Dai H, van de Vijver MJ, et al. Gene expression profiling predicts clinical outcome of breast cancer. Nature. 2002;415(6871):530-536.

38. Creighton CJ, Fu X, Hennessy BT, et al. Proteomic and transcriptomic profiling reveals a link between the PI3K pathway and lower estrogenreceptor (ER) levels and activity in ER+ breast cancer. Breast Cancer Res. 2010;12(3):R40.

39. Creighton CJ, Osborne CK, van de Vijver MJ, et al. Molecular profiles of progesterone receptor loss in human breast tumors. Breast Cancer Res Treat. 2009;114(2):287-299.

40. Goldhirsch A, Wood WC, Coates AS, et al. Strategies for subtypes dealing with the diversity of breast cancer: highlights of the St Gallen International Expert Consensus on the Primary Therapy of Early Breast Cancer 2011. Ann Oncol. 2011;22(8):1736-1747.

41. Osborne CK. Steroid hormone receptors in breast cancer management. Breast Cancer Res Treat. 1998;51(3):227-238.

42. Osborne CK, Neven P, Dirix LY, et al. Gefitinib or placebo in combination with tamoxifen in patients with hormone receptor-positive metastatic breast cancer: a randomized phase II study. Clin Cancer Res. 2011;17(5):1147-1159.

43. Baselga J, Campone M, Piccart M, et al. Everolimus in postmenopausal hormone-receptor-positive advanced breast cancer. $N$ Engl $J$ Med. 2012;366(6):520-529.

44. Casa AJ, Dearth RK, Litzenburger BC, Lee AV, Cui X. The type I insulin-like growth factor receptor pathway: a key player in cancer therapeutic resistance. Front Biosci. 2008;13:3273-3287.

45. Kim HJ, Cui X, Hilsenbeck SG, Lee AV. Progesterone receptor loss correlates with human epidermal growth factor receptor 2 overexpression in estrogen receptor-positive breast cancer. Clin Cancer Res. 2006; 12(3 Pt 2):1013s-1018s.

46. Shou J, Massarweh S, Osborne CK, et al. Mechanisms of tamoxifen resistance: Increased estrogen receptor-HER2/neu cross-talk in ER/HER2-positive breast cancer. $J$ Natl Cancer Inst. 2004;96(12): 926-935.

47. TCGA Data Portal [database on the Internet]. Bethesda (MD): National Cancer Institute. c2007 - [accessed Nov 2010]. Available from: http:// tcga-data.nci.nih.gov/tcga/. 
48. Creighton CJ, Massarweh S, Huang S, et al. Development of resistance to targeted therapies transforms the clinically associated molecular profile subtype of breast tumor xenografts. Cancer Res. 2008;68(18):7493-7501.

49. Miller TW, Hennessy BT, González-Angulo AM, et al. Hyperactivation of phosphatidylinositol-3 kinase promotes escape from hormone dependence in estrogen receptor-positive human breast cancer. J Clin Invest. 2010;120(7):2406-2413.

50. Bayliss J, Hilger A, Vishnu P, Diehl K, El-Ashry D. Reversal of the estrogen receptor negative phenotype in breast cancer and restoration of antiestrogen response. Clin Cancer Res. 2007;13(23):7029-7036.
51. Weigelt B, Hu Z, He X, et al. Molecular portraits and 70-gene prognosis signature are preserved throughout the metastatic process of breast cancer. Cancer Res. 2005;65(20):9155-9158.

52. Abdel-Fatah TM, Powe DG, Hodi Z, Reis-Filho JS, Lee AH, Ellis IO. Morphologic and molecular evolutionary pathways of low nuclear grade invasive breast cancers and their putative precursor lesions: further evidence to support the concept of low nuclear grade breast neoplasia family. Am J Surg Pathol. 2008;32(4):513-523.

\section{Publish your work in this journal}

Biologics: Targets \& Therapy is an international, peer-reviewed journal focusing on the patho-physiological rationale for and clinical application of Biologic agents in the management of autoimmune diseases, cancers or other pathologies where a molecular target can be identified This journal is indexed on PubMed Central, CAS, EMBase, Scopus

Submit your manuscript here: http://www.dovepress.com/biologics-targets--therapy-journal

\section{Dovepress}

and the Elsevier Bibliographic databases. The manuscript management system is completely online and includes a very quick and fair peerreview system, which is all easy to use. Visit http://www.dovepress. com/testimonials.php to read real quotes from published authors. 